\title{
GO-2D: identifying 2-dimensional cellular-localized functional modules in Gene Ontology
}

\author{
Jing Zhu' ${ }^{1}$, Jing Wang ${ }^{1}$, Zheng Guo*1,2, Min Zhang1, Da Yang1, Yanhui Li ${ }^{1}$, \\ Dong Wang ${ }^{1}$ and Guohua Xiao ${ }^{1}$
}

\begin{abstract}
Address: ${ }^{1}$ Department of Bioinformatics, Harbin Medical University, Harbin 150086, China and 2Department of Pharmacology and Biopharmaceutical Key Laboratory of Heilongjiang Province and State, Harbin Medical University, Harbin 150086, China
\end{abstract}

Email: Jing Zhu - jingzhu@ems.hrbmu.edu.cn; Jing Wang - bioccwj@126.com; Zheng Guo* - guoz@ems.hrbmu.edu.cn; Min Zhang - minzhang1982@hotmail.com; Da Yang - yangda1983@126.com; Yanhui Li - liyanhuihb@hotmail.com; Dong Wang - wangdong79@126.com; Guohua Xiao - ghxiao@hrbmu.edu.cn

* Corresponding author

Published: 24 January 2007

BMC Genomics 2007, 8:30 doi:10.1/86/147|-2164-8-30
Received: 4 August 2006

Accepted: 24 January 2007

This article is available from: http://www.biomedcentral.com/I47I-2I64/8/30

(c) 2007 Zhu et al; licensee BioMed Central Ltd.

This is an Open Access article distributed under the terms of the Creative Commons Attribution License (http://creativecommons.org/licenses/by/2.0), which permits unrestricted use, distribution, and reproduction in any medium, provided the original work is properly cited.

\begin{abstract}
Background: Rapid progress in high-throughput biotechnologies (e.g. microarrays) and exponential accumulation of gene functional knowledge make it promising for systematic understanding of complex human diseases at functional modules level. Based on Gene Ontology, a large number of automatic tools have been developed for the functional analysis and biological interpretation of the high-throughput microarray data.

Results: Different from the existing tools such as Onto-Express and FatiGO, we develop a tool named GO-2D for identifying 2-dimensional functional modules based on combined GO categories. For example, it refines biological process categories by sorting their genes into different cellular component categories, and then extracts those combined categories enriched with the interesting genes (e.g., the differentially expressed genes) for identifying the cellular-localized functional modules. Applications of GO-2D to the analyses of two human cancer datasets show that very specific disease-relevant processes can be identified by using cellular location information.

Conclusion: For studying complex human diseases, GO-2D can extract functionally compact and detailed modules such as the cellular-localized ones, characterizing disease-relevant modules in terms of both biological processes and cellular locations. The application results clearly demonstrate that 2-dimensional approach complementary to current I-dimensional approach is powerful for finding modules highly relevant to diseases.
\end{abstract}

\section{Background}

It is widely accepted that functionally related genes tend to express and perform their highly concerted cellular functions in some isolated and interactive modular fashions $[1,2]$. Global gene expression data have provided an opportunity for understanding the transcriptional modularity characterizing complex diseases [3-6]. For example,
Mootha et al. [6] showed that the coordinate disease-associated changes of a set of functionally related genes could be identified even when the expression of individual genes changes modestly. Segal et al. [3] defined 'modules' as gene sets that are conditionally activated or repressed across a wide variety of cancer types, and identified some modules deregulated in cancer. Our recent study demon- 
strated that based on functional modules, i.e., GO categories enriched with differentially expressed genes (DEGs), cancer types can be precisely and robustly classified by supervised classification analysis [5] or discovered by clustering analysis [7].

For high-throughput microarray data analysis, translating lists of interesting genes (e.g., DEGs) into functional modules for understanding the biological phenomena has become an important routine task. Based on Gene Ontology, a large number of tools such as Onto-Express [8], FatiGO [9], GoMiner [10] and GOstat [11] have been developed for this purpose. However, most existing approaches interpret the interesting genes using categories from three ontologies "biological process" (BP), "molecular function" (MF) and "cellular component" (CC) separately, which may be inefficient for mapping some specific modular activities in cells. For example, a GO BP category usually encompasses the genes involved in distinct processes occurring in different cellular compartments [12], and the genes even within a same process may show a clear expression distinction with respect to their cellular localizations [13]. Therefore, in this paper, by combining categories from $\mathrm{BP}, \mathrm{CC}$, and $\mathrm{MF}$, we propose $\mathrm{GO}-2 \mathrm{D}$ as a tool for finding 2-dimensional functional modules (e.g., the cellular-localized modules) for studying complex human diseases.

We use two cancer datasets for numerical analysis, and the results show that with the same FDR (false discovery rate) criteria, many specific processes relevant to diseases cannot be found until additionally cellular location information is used. The results clearly demonstrate the insufficiency of current 1-dimensional approaches and highlight the importance of using 2-dimensional modules for disease analysis.

\section{Implementation}

GO-2D has been implemented in JAVA and interconnected to a relational database system by using MS-Access 2000 for Windows version and SQLite for Linux version.

\section{Database}

In GO-2D, associations of gene IDs from different organisms (including Homo sapiens, Drosophila melanogaster, Caenorhabditis elegans, and Saccharomyces cerevisiae) to GO terms are based on the databases Gene, SGD, FlyBase, and WormBase. Tables relating GO terms with gene IDs can be found in the NCBI web page [14] and GO Consortium web page [15]. The Unigene build \#190 is used in GO-2D.

\section{Analysis and visualization}

Data analysis is made flexible by subdividing the procedure into sequential steps:
(1) Import data: GO-2D starts by reading the input files containing reference and interesting gene lists (see Figure 1). It queries the genes by using Entrez Gene and Unigene for human and organism specific IDs in GO for the other three species (Drosophila melanogaster, Caenorhabditis elegans, and Saccharomyces cerevisiae).

(2) Cross annotation: GO-2D refines a BP category by sorting its genes into different CCs to form combined categories for finding cellular-localized modules enriched with the interesting genes (see Figure 2). It also provides the other 2-dimensional combinations of categories from the three ontologies (BP, MF, and $\mathrm{CC}$ ).

(3) Filter data: GO-2D provides options for finding general or specific combined categories by determining their sizes (the minimum/maximum numbers of included genes) and/or depths in GO.

(4) Statistic test: GO-2D calculates the probability of a combined category having the annotated number of interesting genes by random chance, based on hypergeometric or binomial statistical model [8], which is named "the observed $p$ value".

(5) Multiple tests correction: GO-2D offers Bonferroni correction and FDR control [16] for multiple statistical tests, the results are shown as "the corrected $p$ value". When a total of $n$ combined categories are tested, for the Bonferroni correction, the corrected $p$ value is $p n$, while $p$ is the observed $p$ value. For the FDR control, let $p(k)$ denote the $k$-th smallest observed $p$ value in a total of $n$ combined categories, then the FDR $f_{k}$ for hypothesis $k$ is bounded by $n p(k) / k \leq f_{k}$. If an FDR of $f$ is required for the entire experiment, all hypotheses that satisfy $p(k) \leq f k / n$ are declared as significant. The corrected $p$ value for the $k$ th smallest observed $p$ value is $n p(k) / k$. GO-2D can also output all the observed $p$ values, which can be used for other complicated multiple tests correction by many other existing tools such as the program for Storey's $Q$ value [17].

(6) Results: GO-2D allows users to save the results for detailed examination of the identified modules. The tabular results collect the following information of a combined category: GO IDs, names and depths of categories (e.g. both BP and CC), numbers of genes and interesting genes annotated in it, the observed p values, and the corrected $\mathrm{p}$ values for multiple tests of the combined categories.

(7) Results visualization: GO-2D also provides tree view to visualize the 2-dimensional modules (e.g. BP and CC). GO-2D firstly displays the primary categories (e.g. BP, user defined) in the primary tree, and then in the second- 


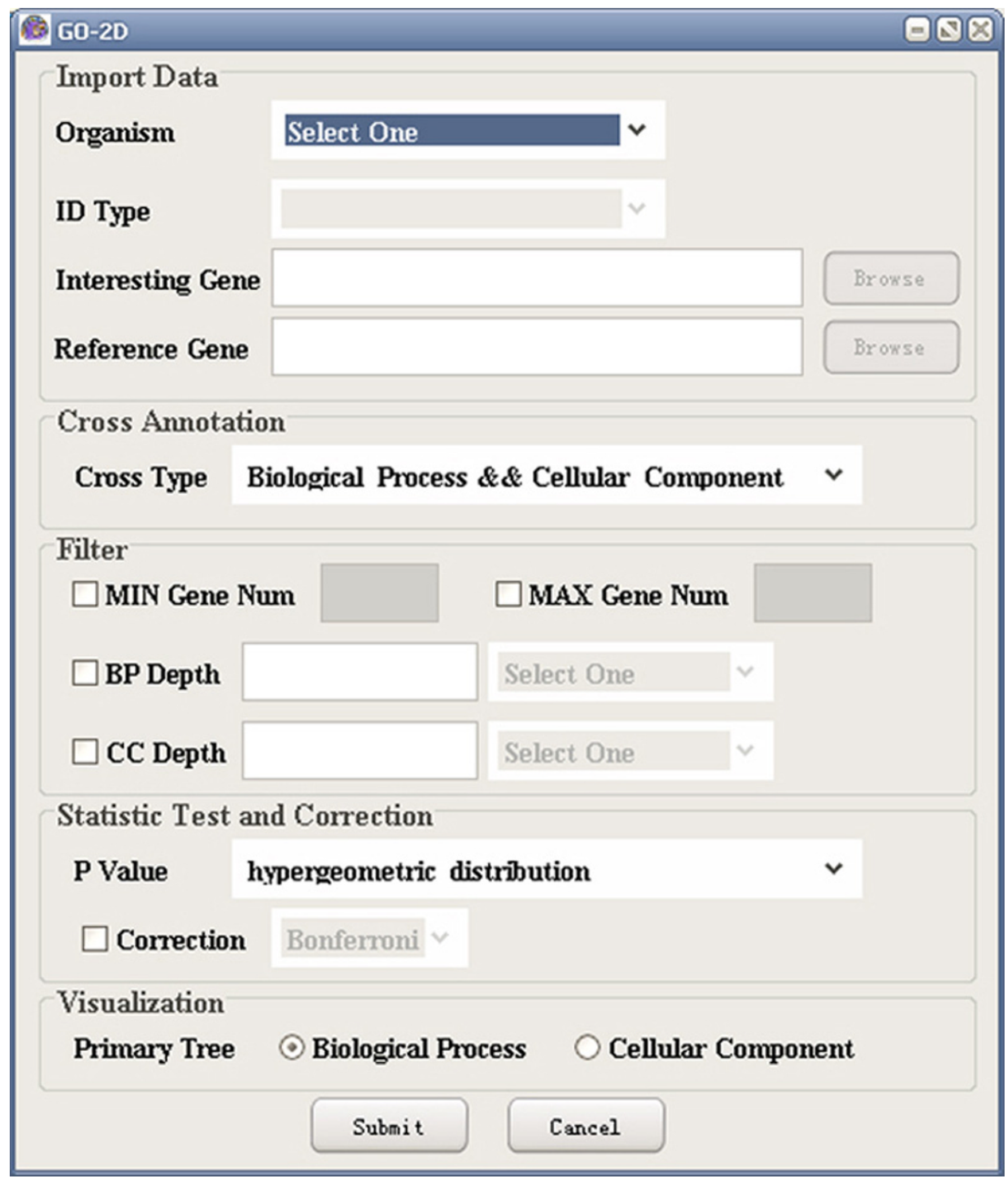

Figure I

A snapshot of GO-2D: the main user interface. 


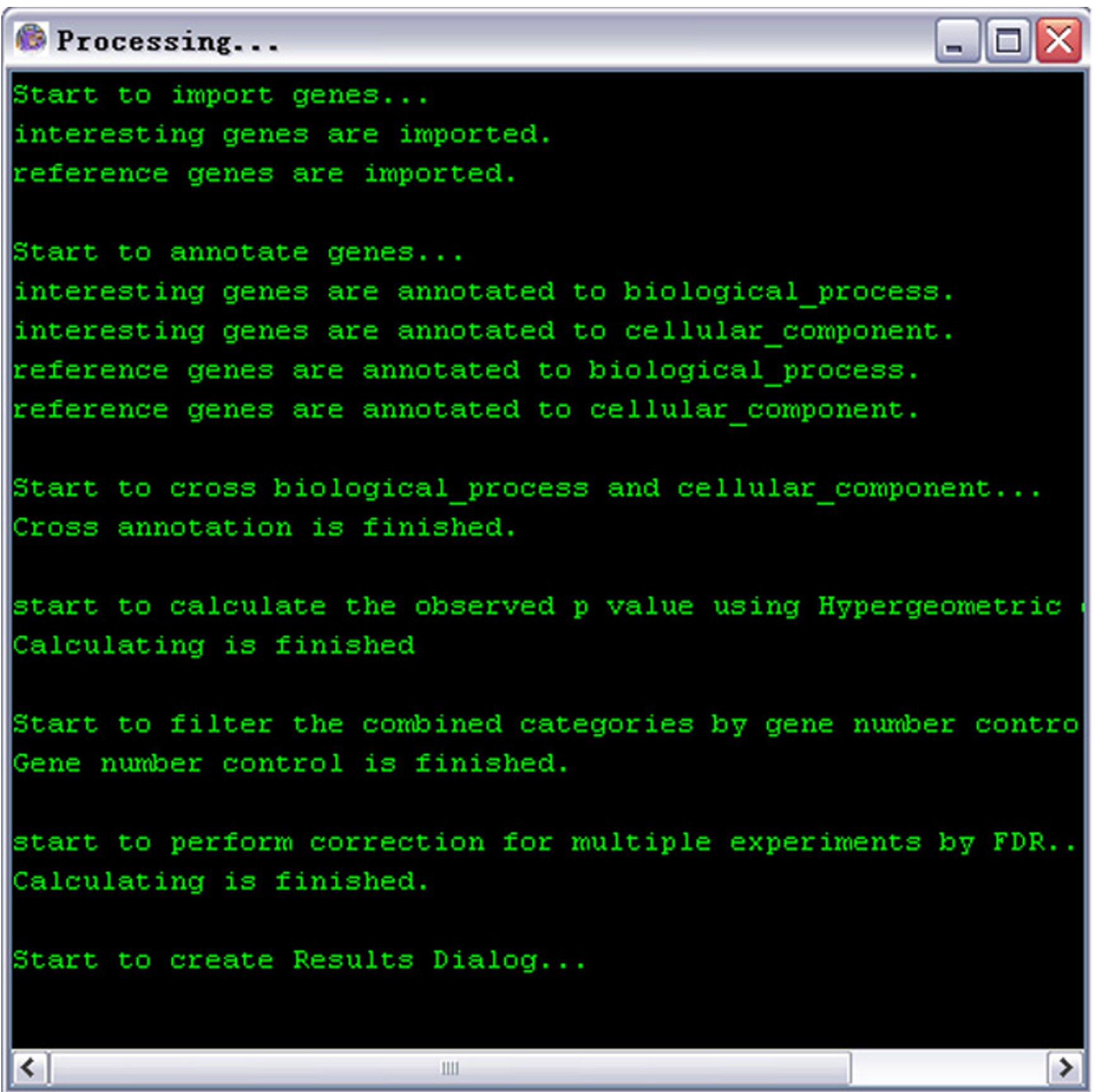

\section{Figure 2}

A snapshot of GO-2D: the processing page.

ary tree, shows the sub-hierarchical structure of the secondary categories (e.g. CC) within each primary category (see Figure 3). The user can select either BP or CC as the "primary tree" for visualization. The selection has no effect on the calculation of over-represented categories.

(8) Redundancy treatment: GO-2D suggests an empirical way to reduce the redundancy among the resulting 2- dimensional modules identified in the hierarchical structure of GO. When some modules share a same primary category in the primary tree (e.g. BP), GO-2D focuses on the combined category containing the most specific secondary category in the secondary tree (e.g. CC).

Details are described in the Additional file 1 (Figure 6, 7, $8,9,10,11,12,13,14,15$-Figure 16). Furthermore, GO- 


\section{Results}

\section{Tree View}

\section{Biological Process Cellular Component Gene Information Undrown Gene}

\section{Gene Ontolozy (Primary Tree)}

$+\cdots$ cell cycle checkpoint CC:15

2 regulation of cyclin dependent protein kinase activity CC:2

$+\cdots$ Glase of mitotic cell cycle CC: 4

G1/S transition of mitotic cell cycle CC:2

W2 phase of mitotic cell cycle CC:1

W2/M transition of mitotic cell cycle CC:2

$+\cdots$ MAPKKK cascade CC:5

+ . $r$ regulation of neurotransmitter levels CC:T

(2) angiogenesis CC: 1

(-). osteoblast differentiation $\mathrm{CC}: 2$

+ . resulation of crtokine oroduction $\mathrm{CC}: 2$

Results Purification

FDR $<=$

\section{Reduce Redundancy}

Detail Choose the combined categories containing one category in primary tree and the most specific categories in the secondary tree.

\section{Save Directory}

\section{Browse}

\section{OK}

\section{Figure 3}

A snapshot of GO-2D: the results page.

2D provides additional standalone software GODAG for visualizing the user selected GO category groups by Directed Acyclic Graph (DAG). In the same DAG, it can show several groups of GO categories marked with different colours, which facilities visual comparisons for the modules identified by different methods (See details in 


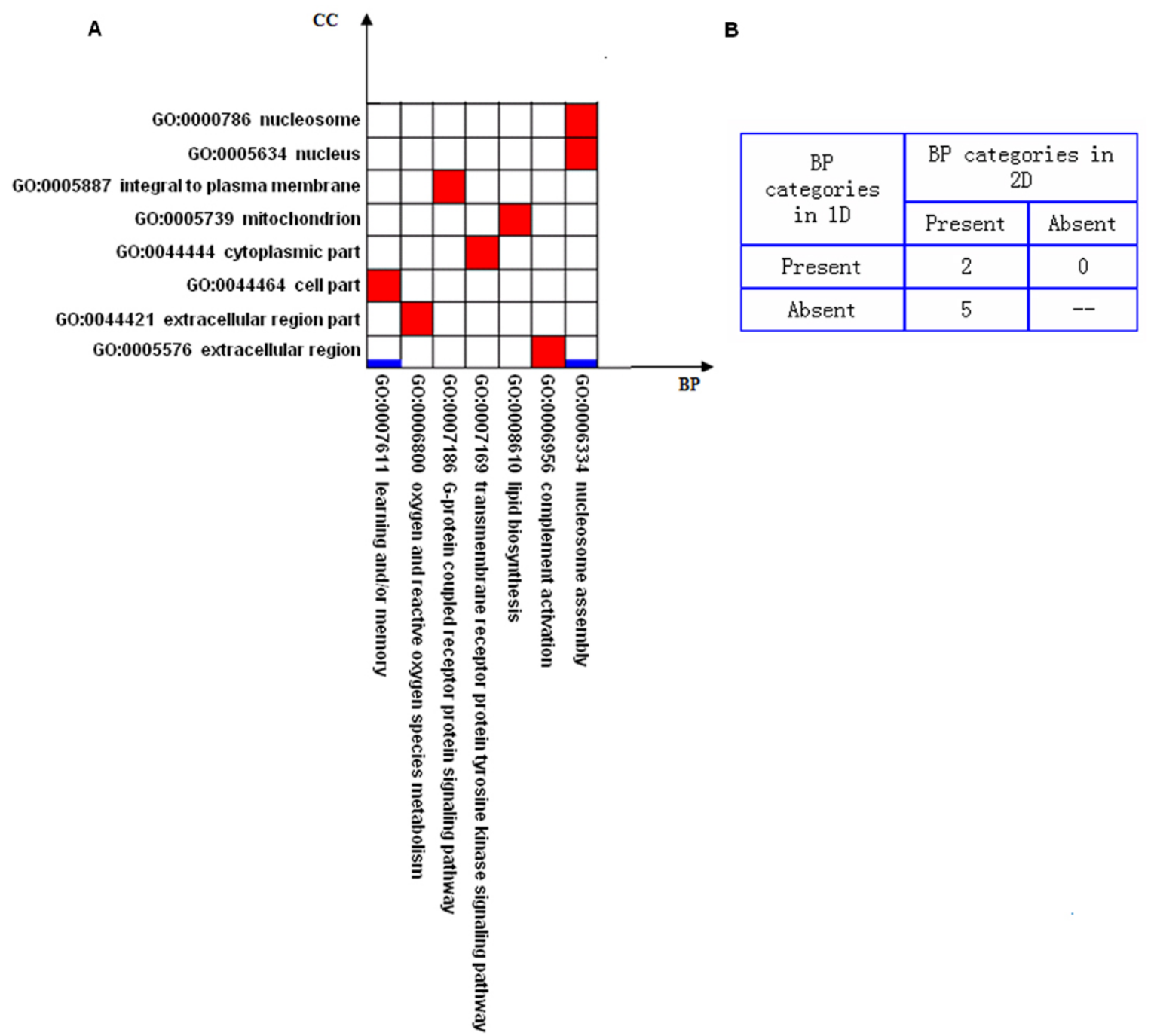

\section{Figure 4}

Comparison between I-dimensional and 2-dimensional modules in breast cancer. (A) Horizontal axis represents BP categories ranked by their depths in the BP ontology. Vertical axis represents CC categories ranked by their depths in the $\mathrm{CC}$ ontology. The thick blue lines represent the I-dimensional modules and the red squares represent the 2-dimensional modules. (B) In the confusion matrix, we show the numbers of BP categories which are present in both I-dimensional and 2-dimensional modules; present in I-dimensional but absent in 2-dimensional modules; absent in I-dimensional but present in 2dimensional modules.

Additional file 2, Figure 17, 18, 19, 20, 21, 22-Figure 23). Also, GO-2D provides another additional standalone tool ConfusionMatrix for comparing the resulting categories identified by 1 - and 2-dimensional approaches in GO-2D (see details in Additional file 3, Figure 24, Figure 25).

\section{Related software comparison}

A recent study [18] has made a detailed comparison of 14 tools for ontological analysis of microarray data. Table 1 compares GO-2D to some typical ones. We highlight that using combined categories for analysis is unique to GO2D.

\section{Results}

Based on the three GO ontologies (Biological_Process, Cellular_Component and Molecular_Function) separately, similar as other tools, GO-2D can also find 1dimensional modules enriched with interesting genes. Because of the multiple tests problem, the observed $p$ value criterion is not justified for comparison, we thus use 


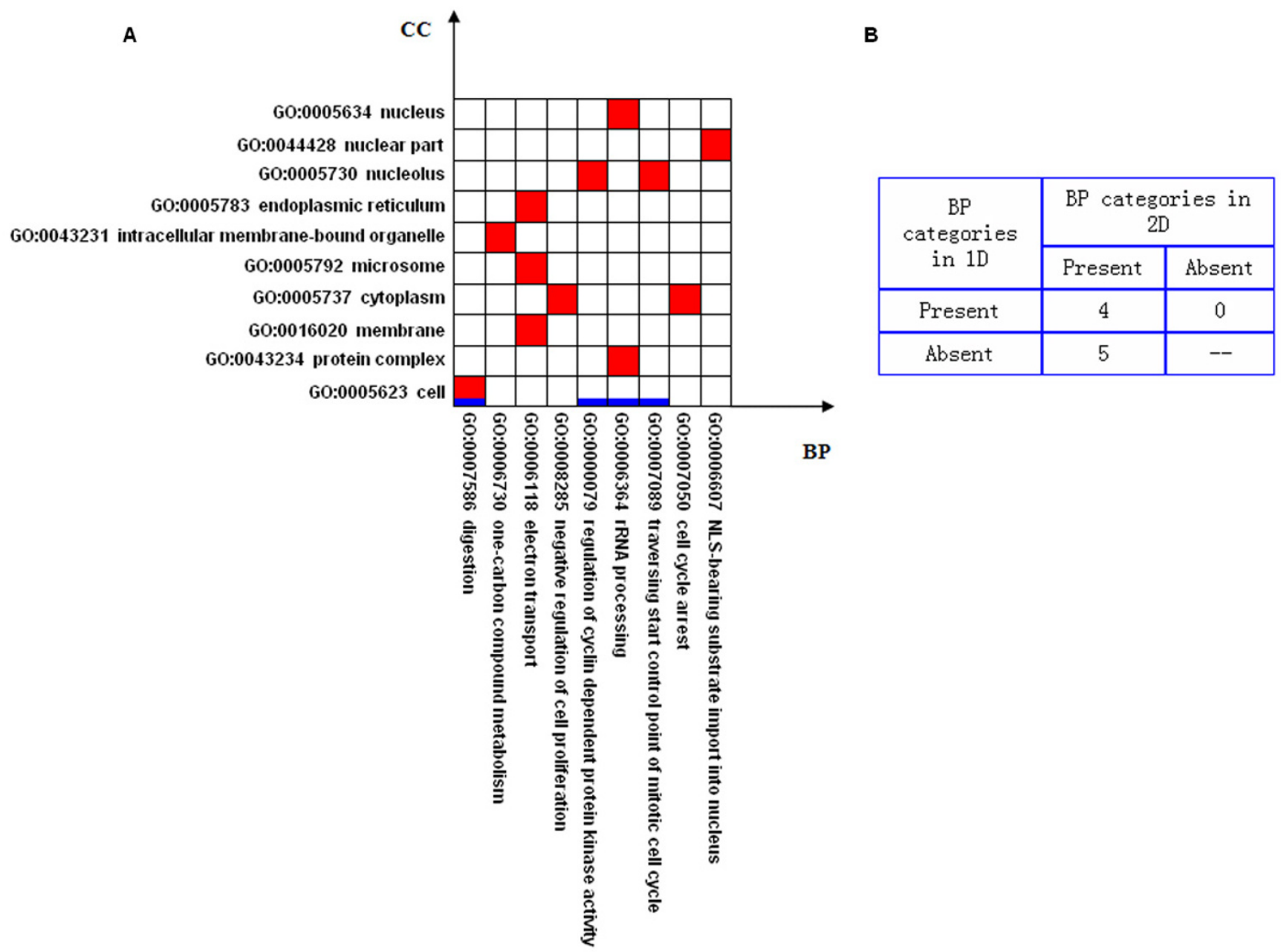

\section{Figure 5}

Comparison between I-dimensional and 2-dimensional modules in gastric cancer. (A) Horizontal axis represents BP categories ranked by their depths in the BP ontology. Vertical axis represents CC categories ranked by their depths in the $\mathrm{CC}$ ontology. The thick blue lines represent the I-dimensional modules and the red squares represent the 2-dimensional modules. (B) In the confusion matrix, we show the numbers of BP categories which are present in both I-dimensional and 2-dimensional modules; present in I-dimensional but absent in 2-dimensional modules; absent in I-dimensional but present in 2dimensional modules.

the same FDR criterion [16] to compare the powers of the approaches to finding 1- and 2-dimensional modules.

\section{Datasets}

The breast cancer dataset contains 20849 genes measured on 21 invasive lobular carcinoma (ILC) and 38 invasive ductal carcinoma (IDC) samples [19]. The gastric cancer dataset contains 20152 genes measured for 103 gastric tumours and 29 normal gastric specimens [20]. Following the pre-processing protocol proposed by Dudoit et al. [21], we eliminate the genes with missing data in more than $5 \%$ arrays, apply a base 2 logarithmic transformation for the remaining expression values, and impute the missing values with zeros. Each experiment is normalized to zero median across the genes. The breast and gastric cancer data finally comprise 8575 and 13037genes (Entrez Gene) respectively, of which 318 and 3388 are differentially expressed genes (DEGs) identified by $t$-test with FDR $1 \%$, calculated by BRB ArrayTools [22].

\section{Parameters}

The parameters are set as following:

(1) Hypergeometric distribution

(2) $\mathrm{FDR}=0.1$ 


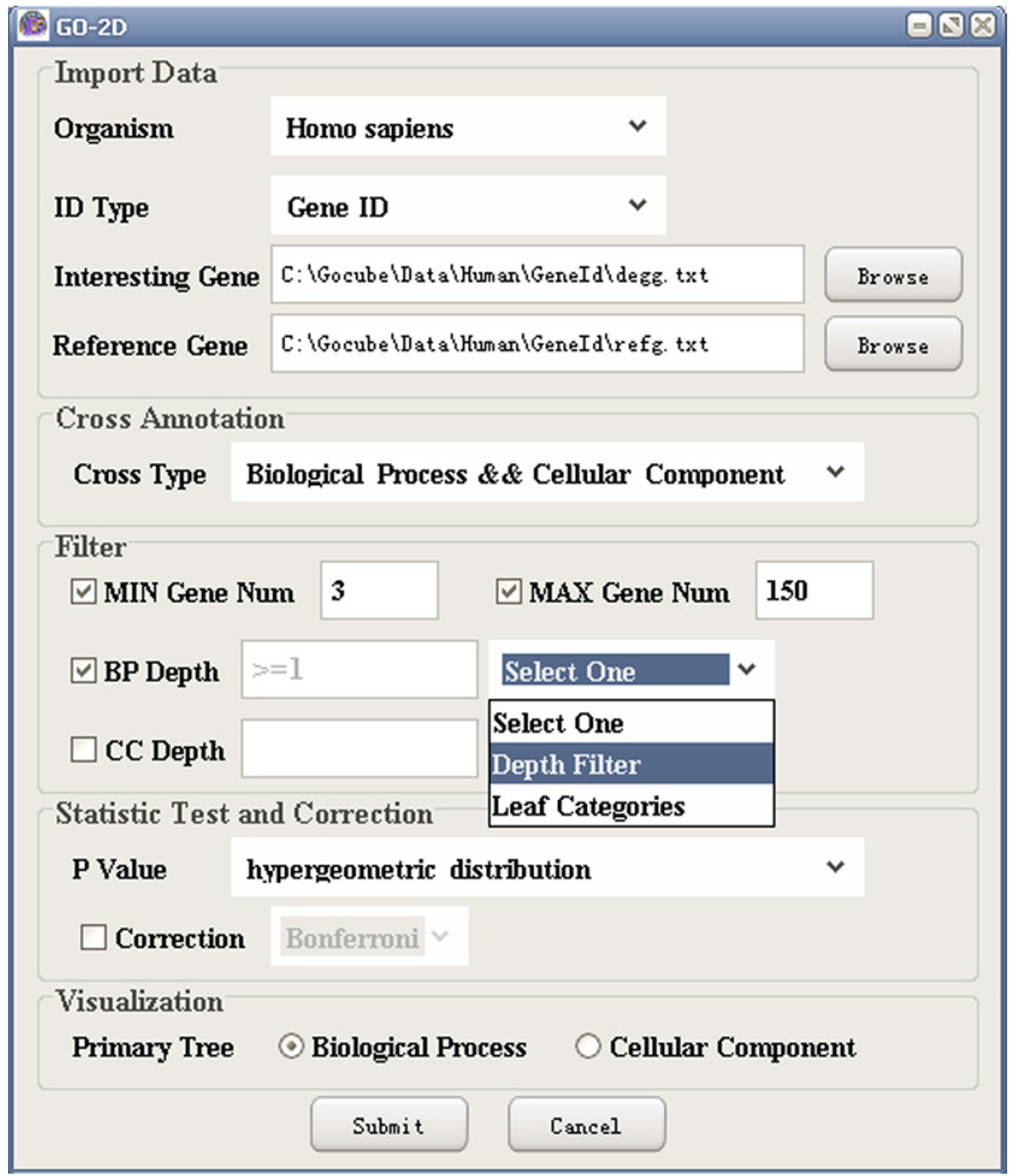

Figure 6

A snapshot of GO-2D: depth selection. 


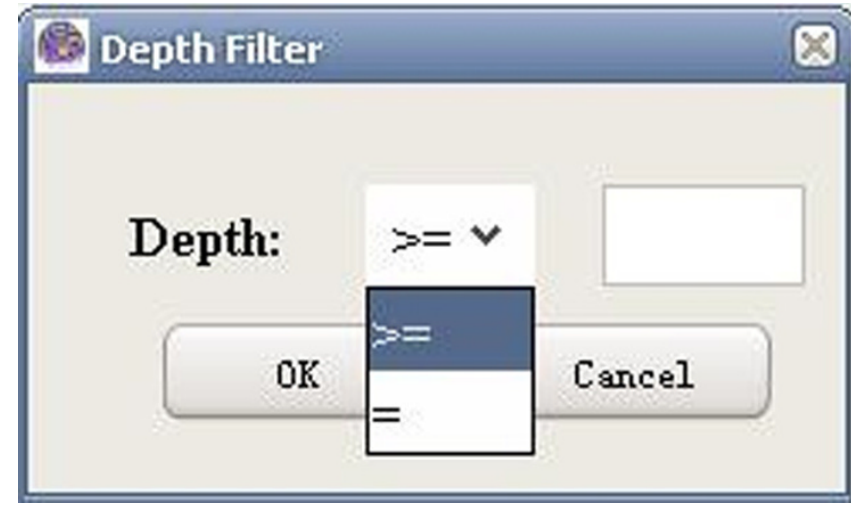

Figure 7

A snapshot of GO-2D: depth filter.
3) MIN Gene Num 3; MAX Gene Num 150

(4) BP depth = Leaf Categories

(5) $\mathrm{CC}$ depth $=1$ (for finding 1-dimensional modules only based on BP), or CC depth>= 1 (for finding 2-dimensional cellular-localized functional modules)

(6) Reduce redundancy

For breast cancer (318 interesting genes and 8575 reference genes), it takes about $9 \mathrm{~min}$ and $12 \mathrm{~min}$ for 1-dimensional and 2-dimensional analysis respectively, with the same computer (CPU: $2.8 \mathrm{GHz}$ and Memory: $1 \mathrm{~GB}$ ). For gastric cancer (3388 interesting genes and 13037 reference genes), it takes about $16 \mathrm{~min}$ and 22 for 1 -dimensional and 2-dimensional analysis, respectively.

\section{Comparison of modules for breast cancer}

With the statistical criterion FDR $\leq 0.1$, we find eight cellular-localized modules, and two 1-dimensional modules

(

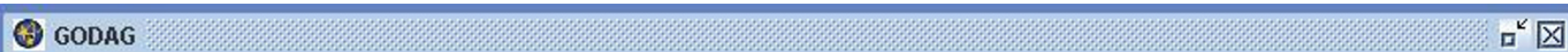

File Edit Help

Group Information

DAG of GO

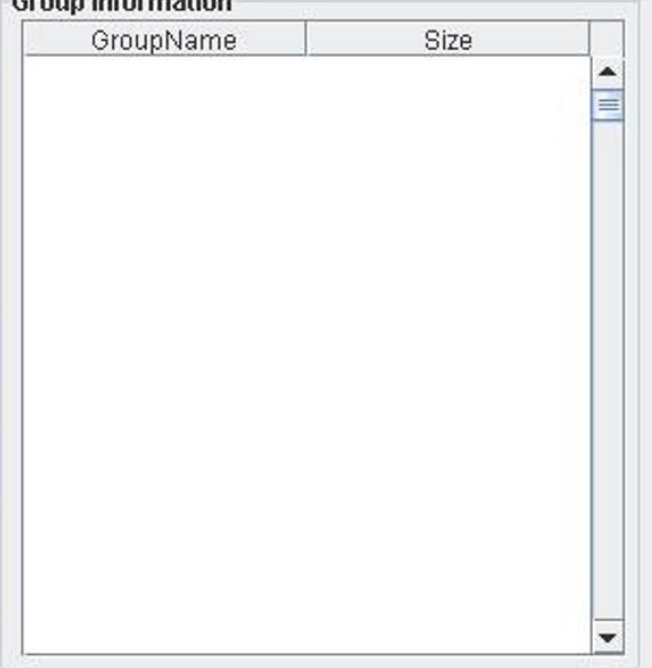

Operation

view Clear

Group Name

GO ID and Name

Figure 17

A snapshot of GODAG: the main user interface. 


\section{Tree View}

\begin{tabular}{|c|lll}
\hline Biological Process Cellular Component Gene Information Urknown Gene \\
\hline
\end{tabular}

Gene Ontolozy (Primary Tree)

+ cell cycle checkpoint CC:15

regulation of cyclin dependent protein kinase activity CC:2

I G1 phase of mitotic cell cycle CC:4

G1/S transition of mitotic cell cycle CC:2

G2 phase of mitotic cell cycle CC:1

S2/M transition of mitotic cell cycle CC:2

+ MAPKKK cascade CC:5

(1) regulation of neurotransmitter levels CC:7

angiogenesis CC:1

(t) osteoblast differentiation CC:2

+ $\cdots$ rezulation of crtokine oroduction $\mathrm{CC}: 2$

\section{Results Purification}

FDR $<=$

\section{Reduce Redundancy}

\section{Detail}

\section{Save Directory}

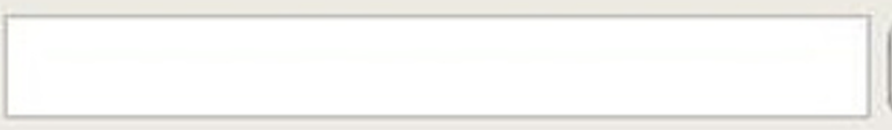

Browse

OK

Figure 8

A snapshot of GO-2D: primary tree view.

based on BP only. As shown in Figure 4 and described in Table 2 (the details of genes in each module are shown in Additional file 4), we can find that, in addition to the bio- logical processes appeared in the two 1-dimensional modules, the 2-dimensional approach discovers some new specific processes relevant to disease. For example, the 


\section{Results}

\section{Tree View}

Biological Process Cellular Component Gene Information Unknown Gene

\section{Gene Ontology (Secondary Tree)}

+. intracellular membrane-bound organelle GENE: 14

+ intracellular non-membrane-bound organelle GENE:9

2. cytoplasmic part GENE:3

+. intracellular organelle part GEME: 10

Results Purification

FDR $<=$

\section{Reduce Redundancy}

\section{Detail}

\section{Save Directory}

\section{Browse}

\section{OK}

\section{Figure 9}

A snapshot of GO-2D: secondary tree view. 


\section{Results}

\section{Tree View}

Biological Process Cellular Component Gene Information Unknown Gene Annotated genes:

1063

1111

11200

2189

4085

4683

5810

675

699

701

Results Purification

FDR $<=$

\section{Reduce Redundancy}

\section{Detail}

Save Directory

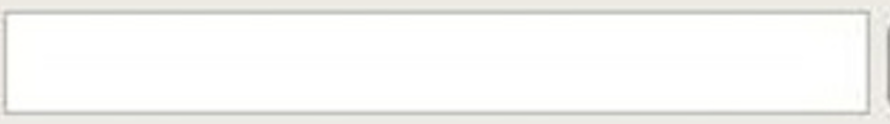

Browse

\section{OK}

Figure 10

A snapshot of GO-2D: gene information (Entrez gene). 


\section{Results}

\section{Tree View}

Biological Process Cellular Component Gene Information Unknown Gene Annotated genes:

Entrez Gene: 1410

UniGene:

Hs. 408767

Entrez Gene: 1915

UniGene:

Hs. 520703

Hs. 522463

Hs. 586423

Hs. 609280

Hs. 613129

Results Purification

FDR $<=$

\section{Reduce Redundancy}

\section{Detail}

\section{Save Directory}

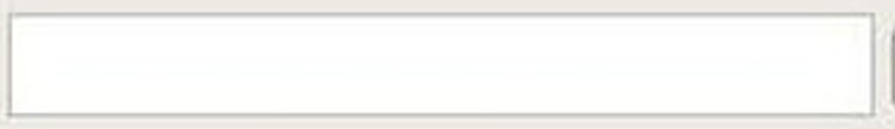

\section{OK}

\section{Figure I I}

A snapshot of GO-2D: gene information (UniGene). 


\section{Results}

\section{Tree View}

Biological Process Cellular Component Gene Information Unknown Gene

Genes without Biological Process annotation 374946

65108

6750

Genes without Cellular Component annotation 374946

65108

\section{Results Purification}

FDR $<=$

\section{Reduce Redundancy}

\section{Detail}

\section{Save Directory}

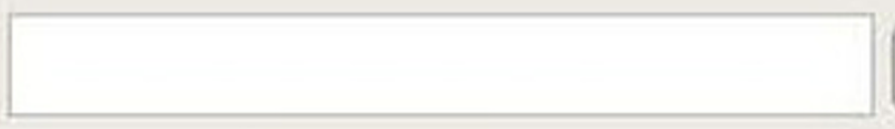

\section{OK}

\section{Figure I 2}

A snapshot of GO-2D: unknown gene (Entrez gene). 


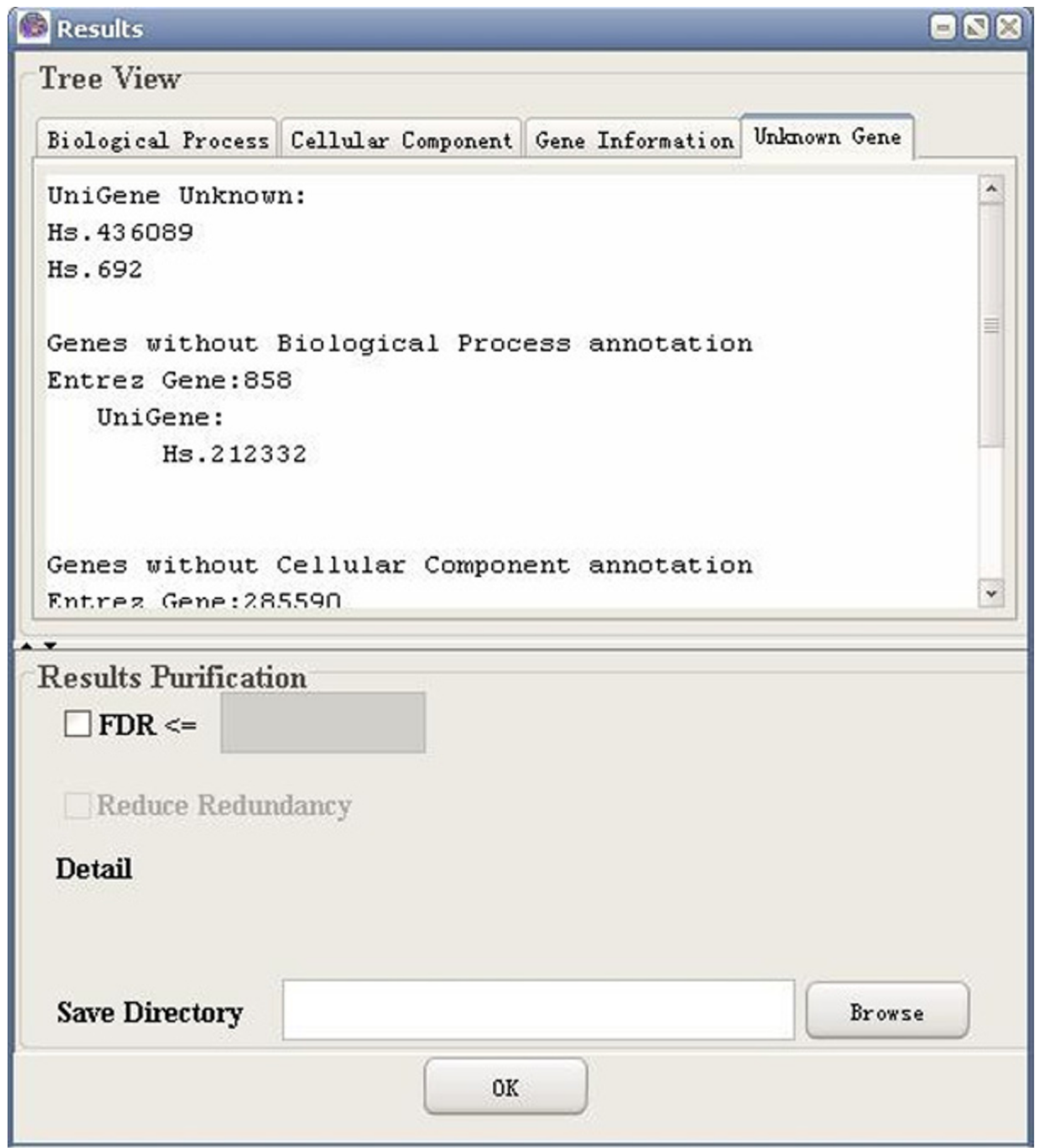

Figure 13

A snapshot of GO-2D: unknown gene (UniGene). 


\section{Results}

\section{Tree View}

\section{Biological Process Cellular Component Gene Information Unknown Gene}

Gene Ontology (Primary Tree)

$+\cdots$ cell cycle checkpoint CC:15

2 regulation of cyclin dependent protein kinase activity CC:2

+ G1 phase of mitotic cell cycle CC:4

G1/S transition of mitotic cell cycle CC:2

G2 phase of mitotic cell cycle CC:1

G2/M transition of mitotic cell cycle CC:2

MAPKKK cascade CC:5

regulation of neurotransmitter levels CC:7

angi ogenesis CC: 1

osteoblast differentiation CC:2

+ . rezulation of cvtokine oroduction $\mathrm{CC}: 2$

Results Purification

FDR $<=$

Reduce Redundancy

Detail

Save Directory 


\section{Results}

\section{Tree View}

\section{Biological Process Cellular Component Gene Information Unknown Gene}

\section{Gene Ontology (Primary Tree)}

$+\cdots$ cell cycle checkpoint CC:15

2. regulation of cyclin dependent protein kinase activity CC:2

+ ... $\mathrm{Gl}$ phase of mitotic cell cycle CC:4

G1/S transition of mitotic cell cycle CC:2

62 phase of mitotic cell cycle CC:1

G2/M transition of mitotic cell cycle CC:2

$+\cdots$ MAPKKK cascade CC:5

+ regulation of neurotransmitter levels CC:T

angi ogenesis CC: 1

$+\cdots$ osteoblast differentiation CC:2

+ -... rezulation of cvtokine production $\mathrm{CC}: 2$

Results Purification

Corrected P Value $<=$

\section{Reduce Redundancy}

\section{Detail}

\section{Save Dinectory}

\section{Browse}

\section{OK}

\section{Figure I5}

A snapshot of GO-2D: corrected p value (bonferroni). 


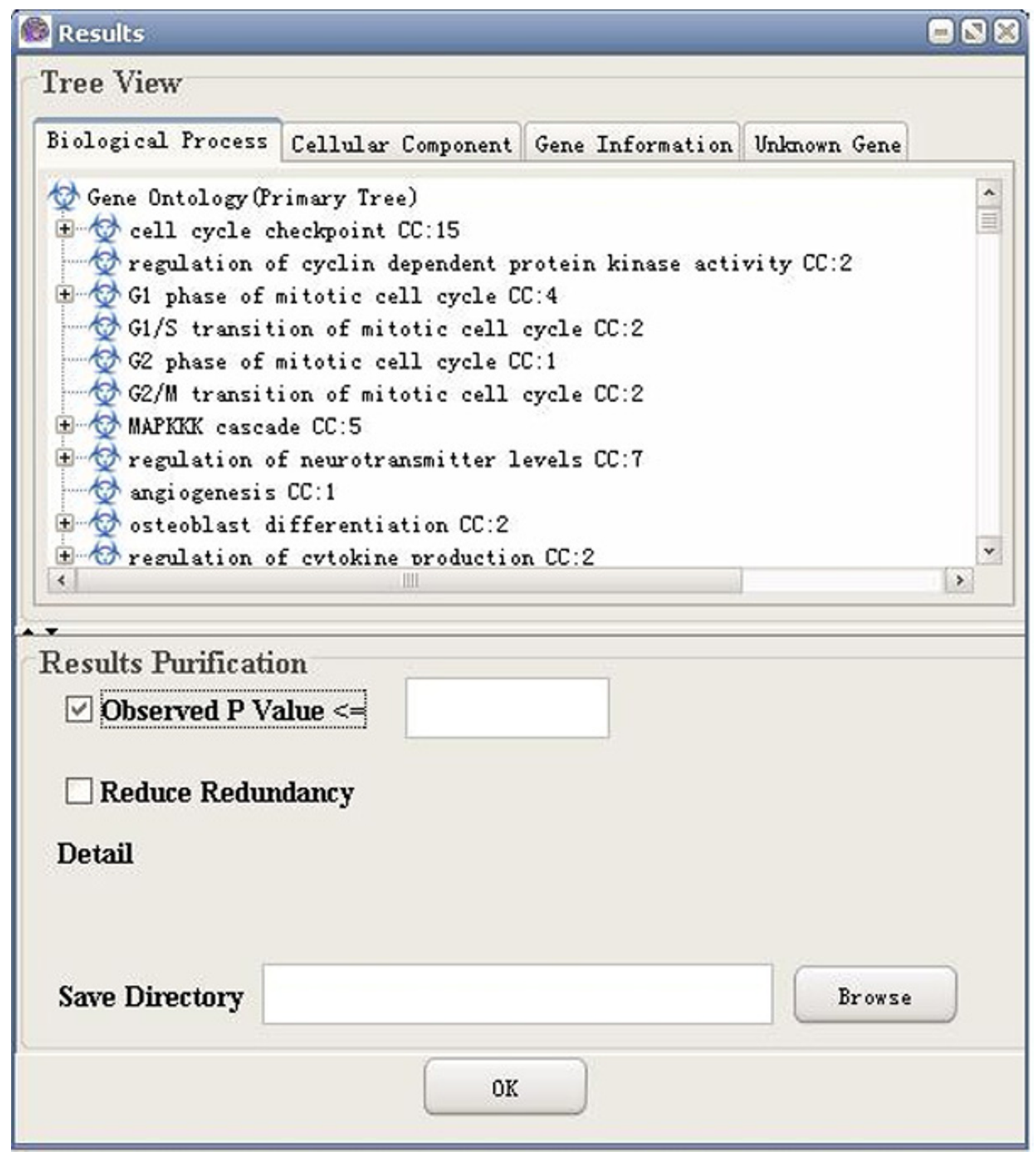

Figure 16

A snapshot of GO-2D: observed $p$ value (no correction is selected). 


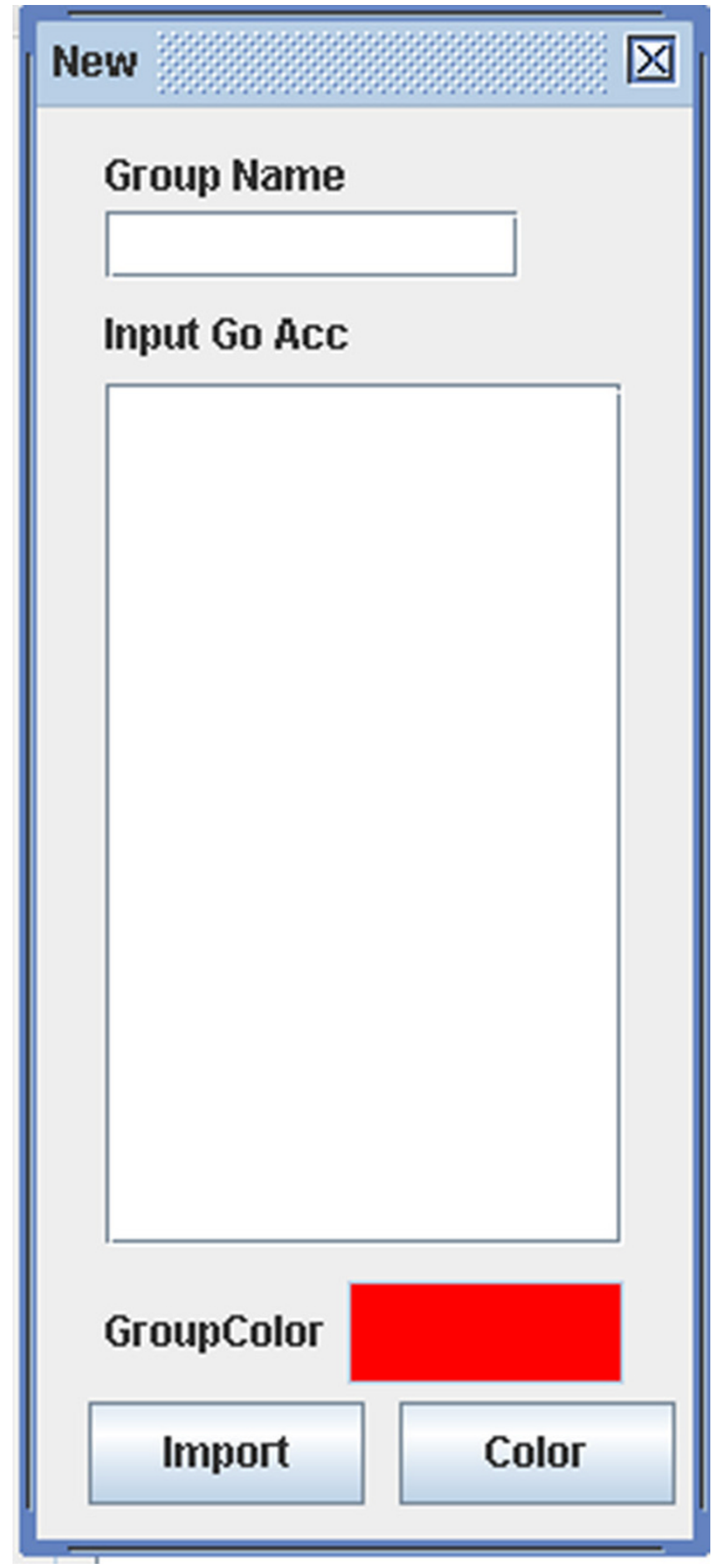

Figure 18

A snapshot of GODAG: input page.

biological process 'lipid biosynthesis' is discovered in the cellular-localized module 'lipid biosynthesis \& mitochondrion'. Using cellular location information, we find that there are three DEGs among the 10 measured genes that are annotated in this cellular-localized module, and the observed p-value is 0.005 (FDR $=4.8 \%)$. However, when we do not use cellular location information, we find four DEGs among the 108 measured genes that are annotated in 'lipid biosynthesis', and the observed p-value is only $0.57(\mathrm{FDR}=65.6 \%)$. This example clearly demonstrates that finding cellular-localized modules is a useful approach to detecting additional disease relevant modules.

A cellular-localized module identified is "BP: oxygen and reactive oxygen species metabolism" in "CC: extracellular region part". Oxidative stress (generating reactive oxygen species) has been linked to cancer initiation and progression. It has been suggested [23] that G. lucidum inhibits the oxidative stress-induced invasive behavior of breast cancer cells by modulating extracellular signal-regulated protein kinases signalling.

For the cellular-localized module "BP: lipid biosynthesis" in "CC: mitochondrial", Zhao, et al. [19] suggested that lipid/fatty acid metabolism may be partially responsible for different proliferation rates of tumor cells in ILCs and IDCs. In addition, mtDNA polymorphisms may be underappreciated factors in breast carcinogenesis [24].

The third example is "BP: G-protein coupled receptor protein signalling pathway" in "CC: integral to plasma membrane", Holland JD et al [25] showed that CXCR4 is subject to controlled regulation in breast cancer cells via differential G protein-receptor complex formation, and this regulation may play a role in the transition from nonmetastatic to malignant tumors.

The last example is for the "BP: complement activation" in "CC: extracellular region". Caragine TA et al. provided direct in vivo evidence that an inhibitor of complement activation can facilitate breast tumor growth by modulating C3 deposition [26].

\section{Comparison of modules for gastric cancer}

With the statistical criterion FDR $\leq 0.1$, we find four 1dimensional modules when based on BP only, and thirteen cellular-localized modules. In addition, as shown in Figure 5 and described in Table 3 (the details of genes in each module are shown in Additional file 4), the 2-dimensional approach detects new disease relevant biological processes combined with the cellular-localization information.

For example, for the cellular-localized functional module "BP: negative regulation of cell proliferation" in "CC: cytoplasm", Li X et al. [27] suggested that TGF-beta1 affects both proliferation and apoptosis of gastric cancer 


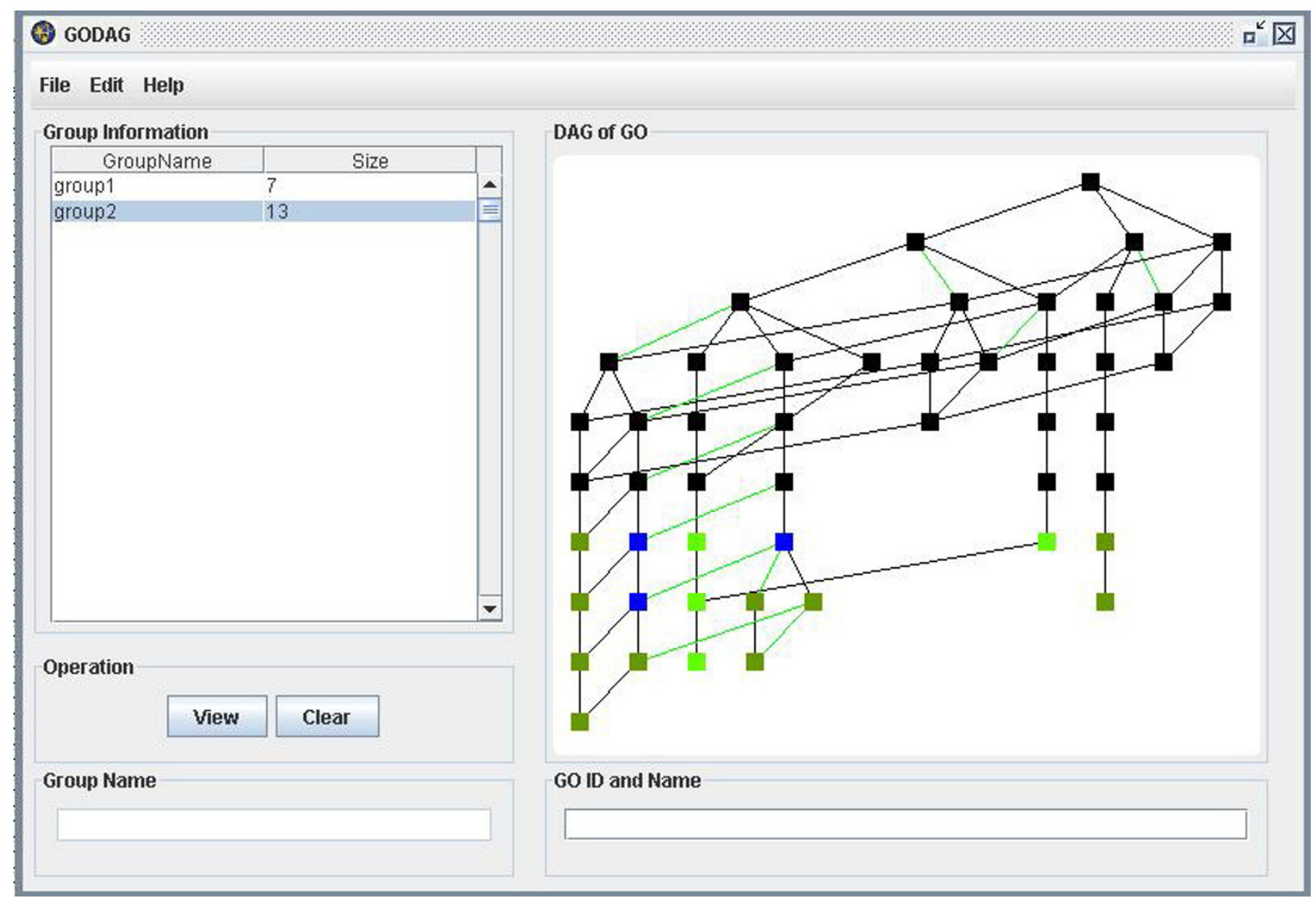

Figure 19

A snapshot of GODAG: result page.

cells through the regulation of p15 and p21, and induces transient expression of Smad 7 as a negative feedback modulation of TGF-beta 1 signal.

Another example is the module "BP: cell cycle arrest" in "CC: cytoplasm". Zheng JY et al. showed that p27 (KIP1) can lead to apoptosis in gastric carcinoma cells [28].

Furthermore, for the same BP, the cellular-localized functional modules are described by additional localization information. For example, for the two cellular-localized modules, "BP: rRNA processing" in "CC: protein complex" and "BP: rRNA processing" in "CC: nucleolus", it has been shown [29] that by reducing the occupancy of the SL1 complex subunits on the rRNA gene promoter and inducing dissociation of the SL1 complex subunits, the transcription of rRNAs is controlled by tumor suppressor PTEN. In addition, a strong correlation has been observed between Nucleolar Organizer regions (loops of DNA encoding ribosomal RNA) counts and metastasis as well as the microscopic type of the gastric carcinoma [30].

\section{Discussion}

When selecting modules from thousands of categories hierarchically structured in GO, the main difficulty is to set statistical significance threshold accounting for the multiplicity of testing. For multiple tests problem, GO-2D adopts the standard methods of Bonferroni correction and FDR control [16,31], which are usually conservative for the non-independent categories organized in ontologies. It has been suggested that re-sampling simulations might be the most reliable way for selecting the significant modules from thousands of categories organized in GO [32]. However, numerical simulations usually suffer from heavy computation burden, and more efficient and feasible re-sampling algorithms deserve further studies [32]. GO-2D outputs the observed p values for the combined categories, which can be used as input data for some more complicated multiple comparisons by existing tools, e.g., the program for Storey's Q value [17].

Since a BP category usually encompasses the genes involved in distinct processes occurring in different cellu- 


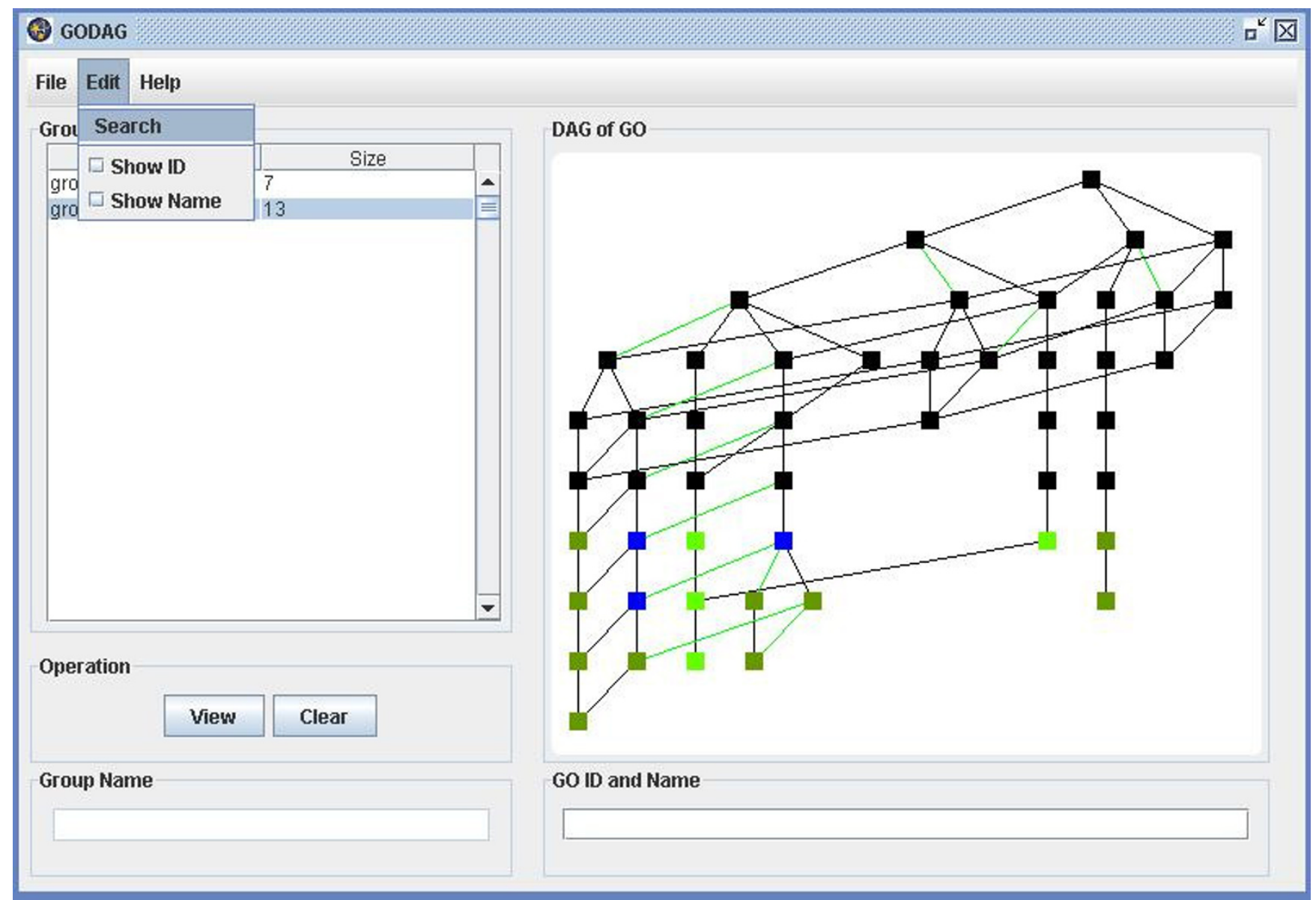

Figure 20

A snapshot of GODAG: edit page.

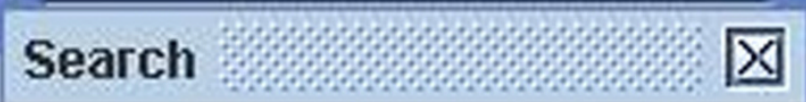

GO ID:

Search

Figure 2 I

A snapshot of GODAG: search page. lar compartments [12] and the genes even within a same process may show a clear expression distinction with respect to their cellular localizations [13], the current 1dimensional approaches are not sufficient enough for identifying the diseases relevant modules. The 2-dimensional approach finds which parts of a BP category, occurring in some cellular compartments, are significantly relevant to disease. As demonstrated by its applications to two cancers in this study, the cellular-localized modules reveal some new biological processes relevant to the diseases in both datasets, in addition to the BPs identified in the 1-dimensional modules. We note that, conceptually, the 2-dimensional approach should cover all BPs identifiable by the 1-dimensional approach, but it might not be always so because of the approximation procedure in the multiple test corrections. Therefore, GO-2D provides both 1- and 2-dimensional approaches for identifying interesting modules of possible disease relevance. When CC Depth is chosen equal to one, the GO-2D just finds only 1-dimensional modules as other software do. Addition- 


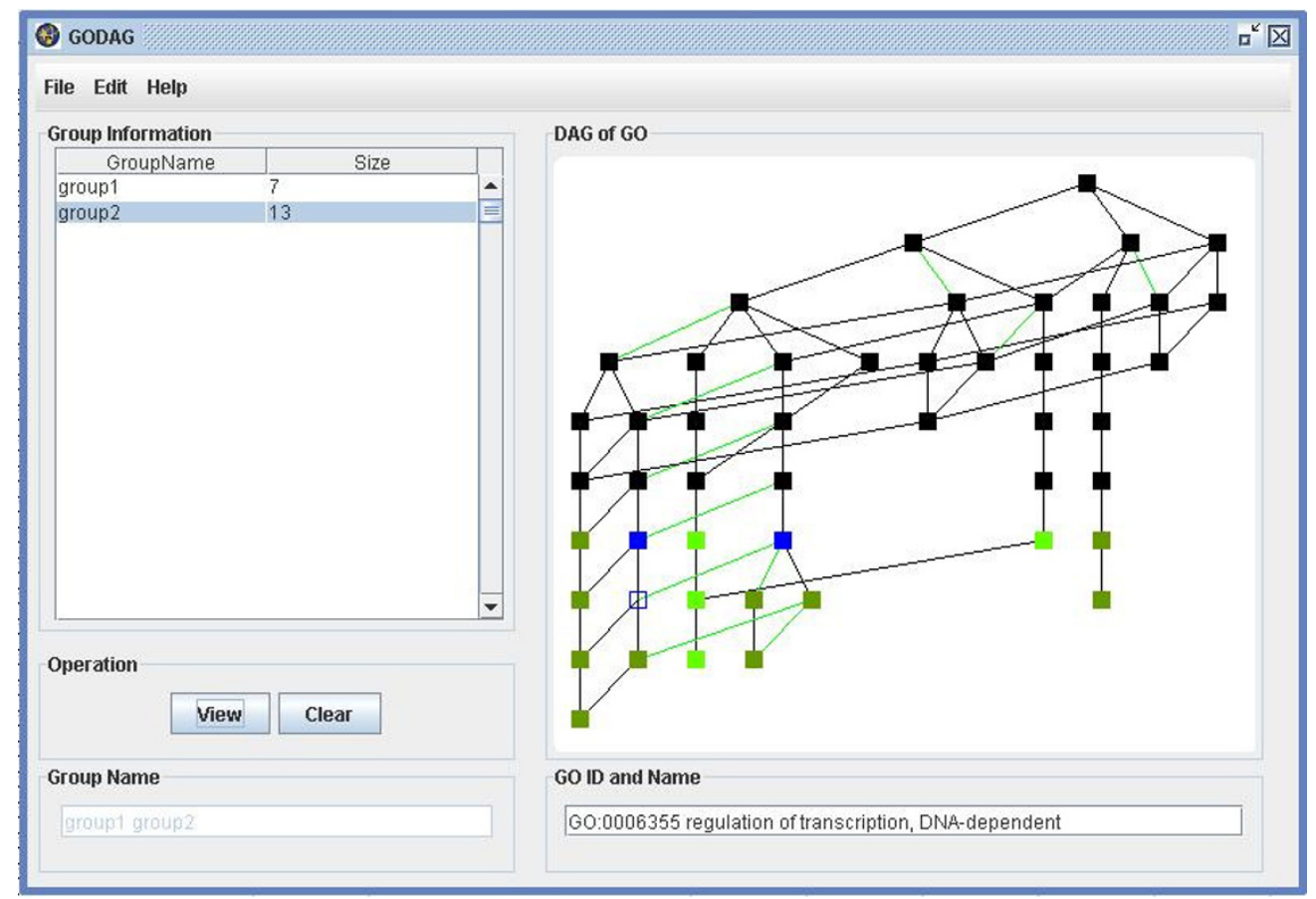

\section{Figure 22}

A snapshot of GODAG: the DAG of resulting categories.

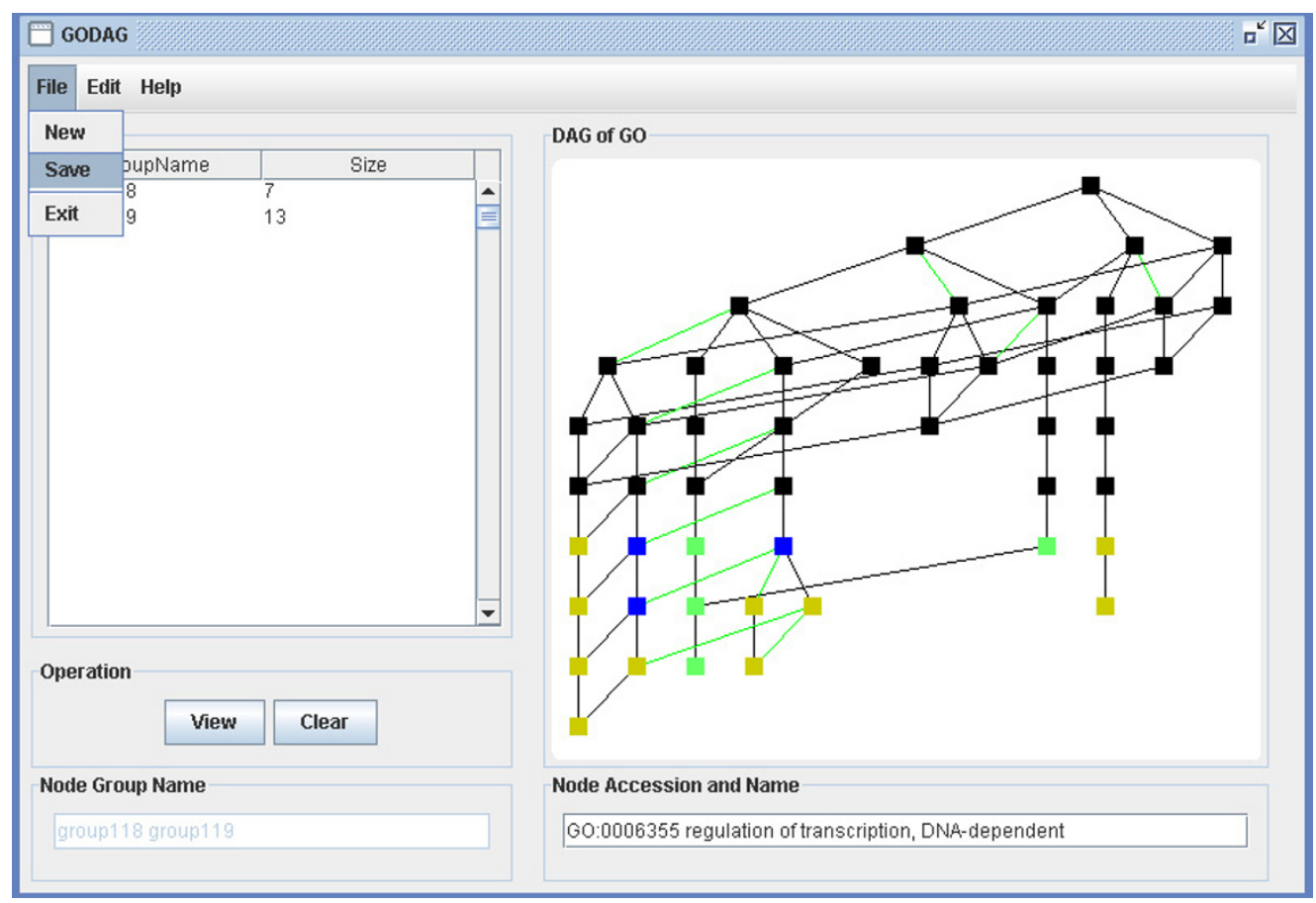

Figure 23

A snapshot of GODAG: save page. 


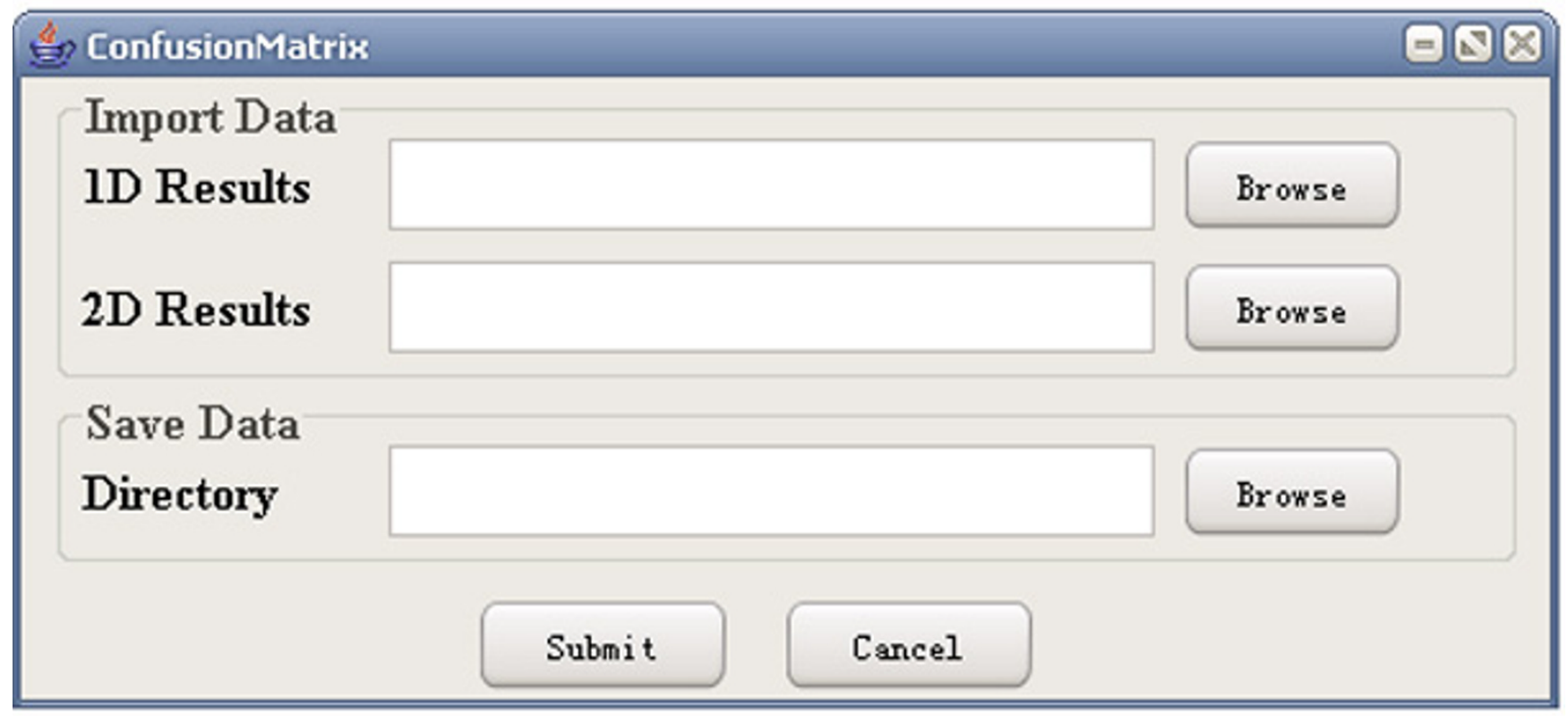

Figure 24

A snapshot of ConfusionMatrix: input page.

ally, GO-2D provides the numbers of genes (from the gene expression dataset) annotated in the original $\mathrm{BP}$ and CC categories of each 2-dimensional module, so the user can filter the results (e.g., according to the overlapping of the original BP and CC categories) to choose their interesting subsets. We conclude that GO-2D is a useful tool of detecting disease relevant modules for one of the most important routine task of the functional analysis and biological interpretation of the high-throughput microarray data.

In a recent study, we have also shown the power of the 2dimensional cellular-localized modules for dissecting the heterogeneity of the complex cancers, i.e. discovering disease subtypes by unsupervised clustering analysis [7]. However, there are still open spaces for further improving the module-based analysis approaches. For example, because changes in gene expression patterns can have various forms, different statistical measures (and their thresholds) for finding DEGs and thus the corresponding functional modules should be further explored. Furthermore, we will integrate GO-2D with more data resources in a future version.

\section{Conclusion}

In summary, we have developed a novel tool for identifying the well-characterized 2-dimensional modules, e.g., in terms of both biological processes and cellular locations. The numerical analyses demonstrate that the 2-dimensional functional modules identified in two cancer datasets enjoy explicit relevance to cancer biology, thus suggesting hints for further experiments confirming the novel modular mechanisms.

\section{Availability and requirements \\ Project Name: GO-2D}

Project home page:

For Windows version: http://www.systembiology.cn/go2d/

For both Windows and Linux version: http:// www.hrbmu.edu.cn/go-2d/index.htm

Operating system(s): Windows 2000 (XP) or Linux

Programming language: Java

Other requirements: Java 1.5

License: GNU General Public License

Restrictions to use by non-academics: Contact corresponding author

\section{Abbreviations}

Gene Ontology (GO), biological process (BP), molecular function (MF), cellular component (CC), differentially expressed genes (DEGs). 


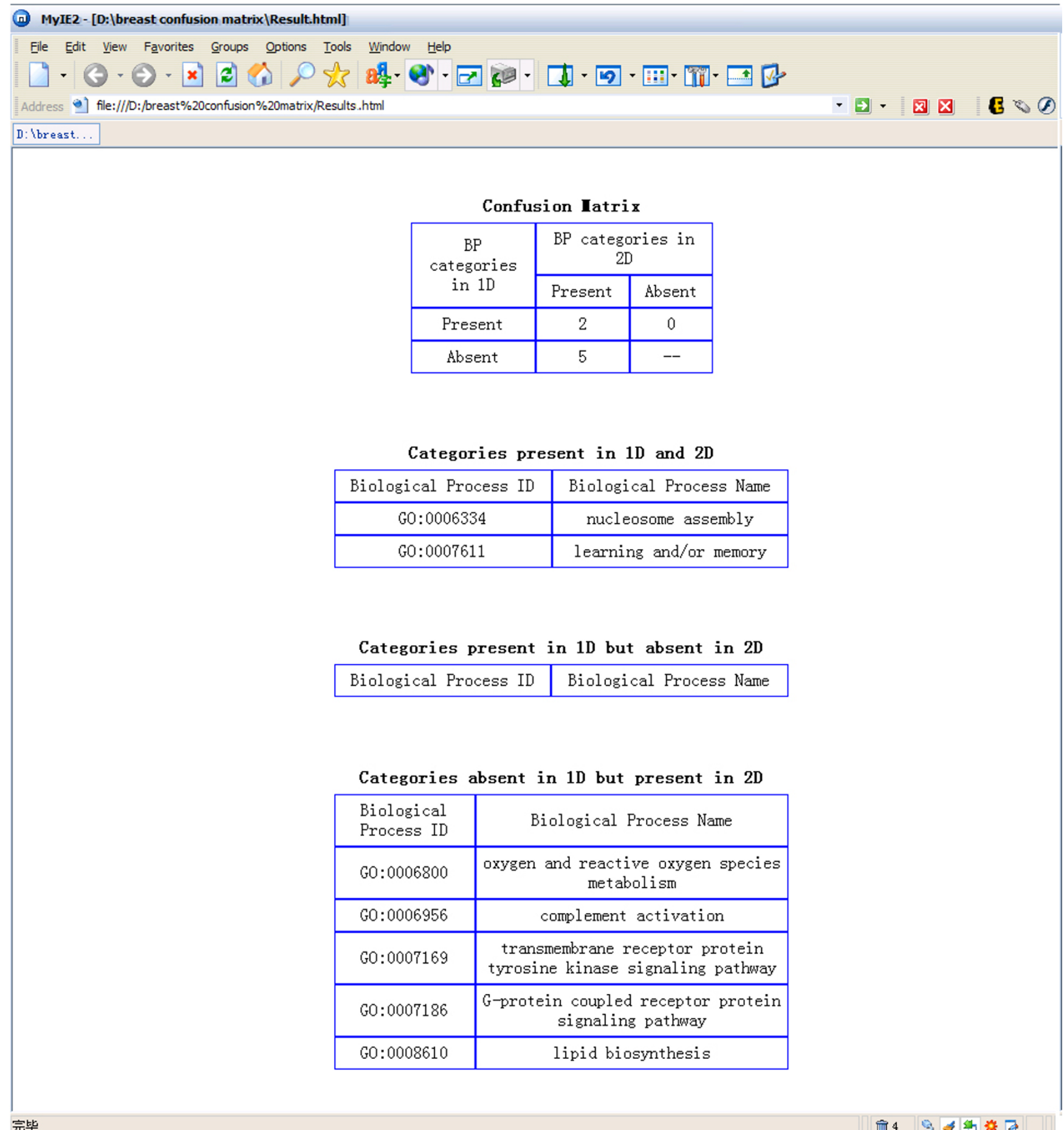

\section{Figure 25}

A snapshot of ConfusionMatrix: results page. 
Table I: Comparison of GO-2D with related software

\begin{tabular}{|c|c|c|c|c|c|}
\hline & Onto-Express & FatiGO & GoMiner & GOstat & GO-2D \\
\hline Analysis scope & 3 single categories & 3 single categories & 3 single categories & 3 single categories & Combined categories \\
\hline Correction for multiple tests & Šidák, Holm, Bonferroni, FDR & Step-down minP, FDR $[\mid 6,31]$ & Relative enrichment & Holm, FDR [16] & Bonferroni, FDR [16] \\
\hline Statistical Analysis & $\begin{array}{l}\text { Hypergeometric, Binomial, } \chi^{2} \text {, } \\
\text { Fisher's exact test, }\end{array}$ & Fisher's exact test & Fisher's exact test & $\chi^{2}$, Fisher's exact test, & Hypergeometric, Binomial \\
\hline Visualization & Flat ${ }^{\mathrm{a}}$, Tree & Flat ${ }^{\mathrm{a}}$, Tree & Tree, DAG & Flat $^{\mathrm{a}}$ & Tree, DAG \\
\hline Application & Web & Web & Stand-alone & Web & Stand-alone \\
\hline
\end{tabular}

Flata: The results are shown without hierarchical structure

Table 2: Functional modules for breast cancer (FDR $\leq \mathbf{0 . 1})$

\begin{tabular}{|c|c|c|c|c|}
\hline Dimension & $\begin{array}{l}\text { Biological Process Name } \\
\text { (\# of genes }{ }^{d} \text { in BP) }\end{array}$ & Cellular Component Name (\# of genes ${ }^{d}$ in CC) & \# of genes ${ }^{d}$ in $2 \mathrm{D}$ module & Observed P Value \\
\hline \multirow[t]{2}{*}{$I D^{b}$} & nucleosome assembly (31) & & & $8.33 \mathrm{E}-04$ \\
\hline & learning and/or memory (7) & --- & --- & $1.58 \mathrm{E}-03$ \\
\hline \multirow[t]{8}{*}{$2 D^{c}$} & nucleosome assembly $(3 \mathrm{I})$ & nucleosome (2I) & 20 & $6.19 \mathrm{E}-05$ \\
\hline & nucleosome assembly (3I) & nucleus (I779) & 31 & $8.33 \mathrm{E}-04$ \\
\hline & learning and/or memory (7) & cell part (4794) & 5 & 4.78E-04 \\
\hline & oxygen and reactive oxygen species metabolism (36) & extracellular region part (257) & 5 & 4.78E-04 \\
\hline & lipid biosynthesis (108) & mitochondrion (376) & 10 & 4.99E-03 \\
\hline & complement activation (14) & extracellular region (388) & II & $6.68 \mathrm{E}-03$ \\
\hline & $\begin{array}{l}\text { transmembrane receptor protein tyrosine kinase } \\
\text { signalling pathway (73) }\end{array}$ & cytoplasmic part (1297) & 11 & $6.68 \mathrm{E}-03$ \\
\hline & $\begin{array}{l}\text { G-protein coupled receptor protein signalling pathway } \\
\text { (I72) }\end{array}$ & integral to plasma membrane (459) & 61 & 7.07E-03 \\
\hline
\end{tabular}

ID: Functional modules based on biological process (one-dimensional modules).

2Dc: Cellular-localized functional modules (two-dimensional modules).

\# of genes ${ }^{d}$ : the numbers of genes from the gene expression dataset (annotated in the original BP/CC categories or 2-dimensional modules)

Table 3: Functional modules for gastric cancer (FDR $\leq \mathbf{0 . 1})$

\begin{tabular}{|c|c|c|c|c|}
\hline Dimension & $\begin{array}{l}\text { Biological Process Name } \\
\text { (\# of genes }{ }^{d} \text { in BP) }\end{array}$ & $\begin{array}{l}\text { Cellular Component Name } \\
\text { (\# of genes }{ }^{d} \text { in CC) }\end{array}$ & \# of genes ${ }^{d}$ in $2 \mathrm{D}$ module & Observed P Value \\
\hline \multirow[t]{4}{*}{$1 D^{b}$} & rRNA processing (46) & & & I.07E-05 \\
\hline & regulation of cyclin dependent protein kinase activity (28) & -- & --- & 2.26E-05 \\
\hline & Digestion (26) & & & $6.04 \mathrm{E}-04$ \\
\hline & traversing start control point of mitotic cell cycle (5) & & & I.18E-03 \\
\hline \multirow[t]{12}{*}{$2 D^{c}$} & rRNA processing (46) & protein complex (I I35) & 22 & $2.22 \mathrm{E}-04$ \\
\hline & rRNA processing (46) & nucleolus (73) & 20 & I.34E-03 \\
\hline & regulation of cyclin dependent protein kinase activity (28) & nucleus (2425) & 18 & 5.27E-05 \\
\hline & Digestion (26) & cell (6889) & 16 & 4.09E-04 \\
\hline & traversing start control point of mitotic cell cycle (5) & nucleus $(2425)$ & 5 & $1.18 \mathrm{E}-03$ \\
\hline & electron transport (229) & endoplasmic reticulum (378) & 49 & I.53E-04 \\
\hline & electron transport (229) & membrane (2978) & 102 & $2.66 \mathrm{E}-04$ \\
\hline & electron transport (229) & microsome (76) & 29 & 7.80E-04 \\
\hline & cell cycle arrest $(50)$ & cytoplasm (2362) & 14 & 4.77E-04 \\
\hline & negative regulation of cell proliferation (107) & cytoplasm (2362) & 37 & $8.4 I E-04$ \\
\hline & NLS-bearing substrate import into nucleus (12) & nuclear part $(465)$ & 5 & I.18E-03 \\
\hline & one-carbon compound metabolism (24) & intracellular membrane-bound organelle (3675) & 7 & 1.67E-03 \\
\hline
\end{tabular}

Note: See the footnotes of Table 2. 


\section{Authors' contributions}

ZG and JZ described and specified the features of, and problems to be solved by GO-2D; JW implemented coding of the software; MZ, DY, YL, DW and GX participated in testing the program and applied the data mining strategy to the field datasets; all authors participated in reading, approving and revising the manuscript.

\section{Additional material}

\section{Additional File 1}

Manual of GO-2D. containing the manual of GO-2D, which is a standalone tool that identifies 2-dimensional functional modules enriched with interesting genes.

Click here for file

[http://www.biomedcentral.com/content/supplementary/14712164-8-30-S1.pdf]

\section{Additional File 2}

Manual of GODAG. containing the manual of GODAG, which is a stand-alone tool that allows the users to visualize their interesting GO categories as a directed acyclic graph.

Click here for file

[http://www.biomedcentral.com/content/supplementary/14712164-8-30-S2.pdf]

\section{Additional File 3}

Manual of ConfusionMatrix. containing the manual of ConfusionMatrix, which is a tool for comparing the resulting categories identified by 1and 2-dimensional approaches in GO-2D.

Click here for file

[http://www.biomedcentral.com/content/supplementary/14712164-8-30-S3.pdf]

\section{Additional File 4}

Gene information. spreadsheet containing the names of all the genes in the modules shown in Table 2 and Table 3.

Click here for file

[http://www.biomedcentral.com/content/supplementary/14712164-8-30-S4.xls]

\section{Acknowledgements}

This work was supported in part by the National Natural Science Foundation of China (Grant Nos. 301705 I5, 30370388), the National High Tech Development Project of China (Grant Nos. 2003AA2Z205I and 2002AA2Z2052).

\section{References}

I. Rives AW, Galitski T: Modular organization of cellular networks. Proc Natl Acad Sci USA 2003, I00(3): I I 28- I I 33.

2. Hartwell LH, Hopfield JJ, Leibler S, Murray AW: From molecular to modular cell biology. Nature 1999, 402(676I Suppl):C47-52.

3. Segal E, Friedman N, Koller D, Regev A: A module map showing conditional activity of expression modules in cancer. Nat Genet 2004, 36(10): 1090-1098.

4. Lamb J, Ramaswamy S, Ford HL, Contreras B, Martinez RV, Kittrell FS, Zahnow CA, Patterson N, Golub TR, Ewen ME: A mechanism of cyclin DI action encoded in the patterns of gene expression in human cancer. Cell 2003, I I 4(3):323-334.
5. Guo Z, Zhang T, Li X, Wang Q, Xu J, Yu H, Zhu J, Wang H, Wang C, Topol EJ, et al:: Towards precise classification of cancers based on robust gene functional expression profiles. BMC Bioinformatics 2005, 6(I):58.

6. Mootha VK, Lindgren CM, Eriksson KF, Subramanian A, Sihag S, Lehar J, Puigserver P, Carlsson E, Ridderstrale M, Laurila E, et al:: PGCIalpha-responsive genes involved in oxidative phosphorylation are coordinately downregulated in human diabetes. Nat Genet 2003, 34(3):267-273

7. Xu JZ, Guo Z, Zhang M, Li X, Li YJ, Rao SQ: Peeling off the hidden genetic heterogeneities of cancers based on disease-relevant functional modules. Mol Med 2006, I 2(I-3):25-33.

8. Khatri P, Bhavsar P, Bawa G, Draghici S: Onto-Tools: an ensemble of web-accessible, ontology-based tools for the functional design and interpretation of high-throughput gene expression experiments. Nucleic Acids Res 2004:W449-456.

9. Al-Shahrour F, Diaz-Uriarte R, Dopazo J: FatiGO: a web tool for finding significant associations of Gene Ontology terms with groups of genes. Bioinformatics 2004, 20(4):578-580.

10. Zeeberg BR, Feng W, Wang G, Wang MD, Fojo AT, Sunshine M, Narasimhan S, Kane DW, Reinhold WC, Lababidi S, et al:: GoMiner: a resource for biological interpretation of genomic and proteomic data. Genome Biol 2003, 4(4):R28.

II. Beissbarth T, Speed TP: GOstat: find statistically overrepresented Gene Ontologies within a group of genes. Bioinformatics 2004, 20(9): I464-| 465.

12. Zhou $X$, Kao MC, Wong WH: Transitive functional annotation by shortest-path analysis of gene expression data. Proc Nat Acad Sci USA 2002, 99(20): 12783-12788.

13. Jimenez JL, Mitchell MP, Sgouros JG: Microarray analysis of orthologous genes: conservation of the translational machinery across species at the sequence and expression level. Genome Biol 2003, 4(I):R4.

14. NCBI Web Page [ftp://ftp.ncbi.nlm.nih.gov/gene/DATA/]

15. GO Consortium [http://www.geneontology.org/]

16. Benjamini $Y, Y H$ : Controlling the false discovery rate: a practical and powerful approach to multiple testing. Journal of the Royal Statistical Society Series B (Methodological) 1995, 57(I):289-300.

17. Storey's $\mathbf{Q}$ Value [http://faculty.washington.edu/jstorey/qvalue]

18. Khatri P, Draghici S: Ontological analysis of gene expression data: current tools, limitations, and open problems. Bioinformatics 2005, 2 I (18):3587-3595.

19. Zhao H, Langerod A, Ji Y, Nowels KW, Nesland JM, Tibshirani R, Bukholm IK, Karesen R, Botstein D, Borresen-Dale AL, et al:: Different gene expression patterns in invasive lobular and ductal carcinomas of the breast. Mol Biol Cell 2004, I 5(6):2523-2536.

20. Chen X, Leung SY, Yuen ST, Chu KM, Ji J, Li R, Chan AS, Law S, Troyanskaya OG, Wong J, et al.: Variation in gene expression patterns in human gastric cancers. Mol Biol Cell 2003, I4(8):3208-32I5.

2I. Dudoit S, Fridlyand J, Speed TP: Comparison of discrimination methods for the classification of tumors using gene expression data. Journal of the American Statistical Association 2002, 97(457):77-87.

22. BRB ArrayTools [http://linus.nci.nih.gov/BRB-ArrayTools.html]

23. Thyagarajan A, Jiang J, Hopf A, Adamec J, Sliva D: Inhibition of oxidative stress-induced invasiveness of cancer cells by Ganoderma lucidum is mediated through the suppression of interleukin-8 secretion. Int J Mol Med 2006, I8(4):657-664.

24. Canter JA, Kallianpur AR, Parl FF, Millikan RC: Mitochondrial DNA GI 0398A polymorphism and invasive breast cancer in African-American women. Cancer Res 2005, 65(7):8028-8033.

25. Holland JD, Kochetkova M, Akekawatchai C, Dottore M, Lopez A, McColl SR: Differential functional activation of chemokine receptor CXCR4 is mediated by $\mathbf{G}$ proteins in breast cancer cells. Cancer Res 2006, 66(8):41117-4I24.

26. Caragine TA, Okada N, Frey AB, Tomlinson S: A tumor-expressed inhibitor of the early but not late complement lytic pathway enhances tumor growth in a rat model of human breast cancer. Cancer Res 2002, 62(4): III0-IIII.

27. Li X, Zhang YY, Wang Q, Fu SB: Association between endogenous gene expression and growth regulation induced by TGF-beta I in human gastric cancer cells. World J Gastroenterol 2005, II (I):6I-68. 
28. Zheng JY, Wang WZ, Li KZ, Guan WX, Yan W: Effect of p27(KIPI) on cell cycle and apoptosis in gastric cancer cells. World J Gastroenterol 2005, I I (45):7072-7077.

29. Zhang C, Comai L, Johnson DL: PTEN represses RNA Polymerase I transcription by disrupting the SLI complex. Mol Cell Biol 2005, 25(16):6899-69II.

30. Prakash I, Mathur RP, Kar P, Ranga S, Talib VH: Comparative evaluation of cell proliferative indices and epidermal growth factor receptor expression in gastric carcinoma. Indian J Pathol Microbiol 1997, 40(4):48I-490.

31. Benjamini Y, Drai D, Elmer G, Kafkafi N, I G: Controlling the false discovery rate in behavior genetics research. Behav Brain Res 200I, I 25(I-2):279-284.

32. Osier MV, Zhao H, Cheung KH: Handling multiple testing while interpreting microarrays with the Gene Ontology Database. BMC Bioinformatics 2004, 5(I): 124.

Publish with Biomed Central and every scientist can read your work free of charge

"BioMed Central will be the most significant development for disseminating the results of biomedical research in our lifetime. "

Sir Paul Nurse, Cancer Research UK

Your research papers will be:

- available free of charge to the entire biomedical community

- peer reviewed and published immediately upon acceptance

- cited in PubMed and archived on PubMed Central

- yours - you keep the copyright

Submit your manuscript here:

http://www.biomedcentral.com/info/publishing_adv.asp
BioMedcentral 